\title{
ON THE MODULE STRUCTURE OF A GROUP ACTION ON A LIE ALGEBRA
}

\author{
ATHANASSIOS I. PAPISTAS
}

(Received 2 February 2005; revised 1 November 2005)

\author{
Communicated by D. Easdown
}

\begin{abstract}
Let $G$ be a finite group, $K$ a field, and $V$ a finite-dimensional $K G$-module. Write $L(V)$ for the free Lie algebra on $V$; similarly, let $M(V)$ be the free metabelian Lie algebra. The action of $G$ extends naturally to these algebras, so they become $K G$-modules, which are direct sums of finite-dimensional submodules. This paper explores whether indecomposable direct summands of such a $K G$-module (for some specific choices of $G, K$ and $V$ ) must fall into finitely many isomorphism classes. Of course this is not a question unless there exist infinitely many isomorphism classes of indecomposable $K G$-modules (that is, $K$ has positive characteristic $p$ and the Sylow $p$-subgroups of $G$ are non-cyclic) and $\operatorname{dim} V>1$.

The first two results show that the answer is positive for $M(V)$ when $K$ is finite and $\operatorname{dim} V=2$, but negative when $G$ is the Klein four-group, the characteristic of $K$ is 2, and $V$ is the unique 3-dimensional submodule of the regular module $D$. In the third result, $G$ is again the Klein four-group, $K$ is any field of characteristic 2 with more than 2 elements, $V$ is any faithful module of dimension 2 , and $B$ is the unique 3-dimensional quotient of $D$; the answer is positive for $L(V)$ if and only if it is positive for each of $L(B)$, $L(D)$, and $L(V \otimes V)$.
\end{abstract}

2000 Mathematics subject classification: primary 17B01, 17B30; secondary $20 \mathrm{C} 20$.

\section{Introduction and notation}

Let $K$ be a field of positive characteristic $p$ and $G$ a group. Throughout this paper, all $K G$-modules considered will be right $K G$-modules and all tensor products are tensor products over $K$. If $V$ is a vector space over $K$ (or, briefly, $K$-space), we write $A(V)$ for the free associative algebra (with identity element) on $V$ : thus $A(V)$ is the free associative algebra over $K$ with the property that $A(V)$ contains $V$ as a subspace, and every basis for $V$ generates $A(V)$ freely as an algebra. If $V$ is a $K G$-module, the

(c) 2007 Australian Mathematical Society $1446-7887 / 07 \$ A 2.00+0.00$ 
action of $G$ on $V$ extends uniquely to $A(V)$ subject to $A(V)$ becoming a $K G$-module on which the elements of $G$ act as algebra automorphisms. Similarly, $R(V)$ denotes the free restricted Lie algebra on $V$ and $L(V)$ denotes the free Lie algebra on $V$. It is well-known that if $A(V)$ is regarded as a restricted Lie algebra under the operations given by $[a, b]=a b-b a$ and $a^{[p]}=a^{p}$, then the Lie subalgebra generated by $V$ and the restricted Lie subalgebra generated by $V$ may be identified with $L(V)$ and $R(V)$, respectively. In this sense, we consider $L(V)$ and $R(V)$ submodules of $A(V)$. For a non-negative integer $n$, let $A^{n}(V)$ be the $n$th homogeneous component of $A(V)$ : it is the subspace of $A(V)$ spanned by all monomials $v_{1} \ldots v_{n}$, with $v_{1}, \ldots, v_{n} \in V$, and each $A^{n}(V)$ is a $K G$-module. It is well-known that $A(V)$ has the following direct decomposition as $K$-space: $A(V)=\bigoplus_{n \geq 0} A^{n}(V)$ with $A^{0}(V)=K$. For $n \geq 1$, the homogeneous components of degree $n$ in $L(V)$ and $R(V)$ are given by $L^{n}(V)=L(V) \cap A^{n}(V)$ and $R^{n}(V)=R(V) \cap A^{n}(V)$. The free metabelian Lie algebra on $V$ is defined by $M(V)=L(V) / L(V)^{\prime \prime}$, where $L(V)^{\prime \prime}$ is the second derived algebra of $L(V)$. Furthermore, we write $S(V)$ for the symmetric algebra on $V$. These algebras will be regarded as $K G$-modules in the obvious way and their homogeneous components of degree $n$ will be denoted by $M^{n}(V)$ (for $n \geq 1$ ) and $S^{n}(V)$ (for $n \geq 0$, and $\left.S^{0}(V)=K\right)$, respectively. Each $M^{n}(V)$ and $S^{n}(V)$ is a $K G$-module. For any Lie algebra $L$ over $K$ (or, briefly, Lie algebra), we write $[u, v]$ for the Lie product with $u, v \in L$, and expressions of the form $\left[u_{1}, u_{2}, \ldots, u_{n}\right]$ are taken as left-normed so that $\left[u_{1}, u_{2}, \ldots, u_{n}\right]=\left[\left[u_{1}, \ldots, u_{n-1}\right], u_{n}\right]$ for $n \geq 3$.

For a finite-dimensional $K G$-module $V$, let $\mathscr{I}(V)$ denote the set of isomorphism classes of indecomposable direct summands of $V$. A graded $K G$-module is a $K G$-module $V$ with a distinguished decomposition $V=V_{1} \oplus V_{2} \oplus \cdots$ where each $V_{n}$ is a finite-dimensional $K G$-module. For a graded $K G$-module $V$, we define $\mathscr{I}(V)=\bigcup_{n \geq 1} \mathscr{I}\left(V_{n}\right)$. All the aforementioned algebras on a finite-dimensional $K G$-module are graded $K G$-modules. By a result of Higman (see [10, Chapter VII, Theorem 5.4]), a necessary and sufficient condition for the existence of infinitely many isomorphism classes of indecomposable $K G$-modules is that the field $K$ has positive characteristic $p$ and the group $G$ has a non-cyclic Sylow $p$-subgroup.

Our main purpose in this paper is to study whether $\mathscr{I}(L(V))$ or $\mathscr{I}(M(V))$ is finite for certain $K, G$ and $V$. Let $K$ be a finite field, $G=\operatorname{SL}(2, K)$ and $V$ the natural 2-dimensional $K G$-module. It follows from a result of Alperin and Kovács [1] that $\mathscr{I}(S(V))$ is finite. In Section 2, we prove the analogous result for the free metabelian Lie algebra $M(V)$ (see Proposition 2.1). Karagueuzian and Symonds (see [11]) generalized the aforementioned result for symmetric algebras as follows: for any finite group $G$ (not necessarily a p-group) and any $K G$-module with $\operatorname{dim}_{K} V \leq 3, \mathscr{I}(S(V)$ ) is finite. In the case of the free metabelian Lie algebra $M(V)$, with $\operatorname{dim}_{K} V=3$ and $K$ a field of characteristic 2, the situation is different. In particular, let $U_{3}(K)$ be the group of $3 \times 3$ upper unitriangular matrices over $K$ and let $V$ be the natural 
3-dimensional $K U_{3}(K)$-module. Then $\mathscr{I}(M(V))$ is not finite (see Corollary 2.6).

In Section 3, we consider free Lie algebras. For any finite-dimensional $V$, the Lie subalgebra of $R(V)$ generated by $R^{2}(V) \oplus R^{3}(V)$ is freely generated by this submodule, so we may denote it by $L\left(R^{2}(V) \oplus R^{3}(V)\right)$. Write

$$
L_{\text {grad }}^{n}\left(R^{2}(V) \oplus R^{3}(V)\right)=R^{n}(V) \cap L\left(R^{2}(V) \oplus R^{3}(V)\right)
$$

for its $n$th homogeneous component in the grading it inherits from $R(V)$. We prove that if $K$ is any field of characteristic $2, G$ is any group, $V$ is any 2-dimensional $K G$-module, and $n \geq 3$, then $L^{n}(V)=L_{\text {grad }}^{n}\left(R^{2}(V) \oplus R^{3}(V)\right)$. This fact turns out to be very useful in the case when $K$ has more than 2 elements, $G$ is the Klein four-group, and $C$ is any faithful 2-dimensional $K G$-module, for then it enables us to show that $\mathscr{I}(L(C))$ is finite if and only if $\mathscr{I}(L(D)), \mathscr{I}(L(B))$ and $\mathscr{I}(L(C \otimes C))$ are finite, where $D$ is the regular $K G$-module and $B$ is the unique 3-dimensional quotient of $D$. We note that Michos has given an example of a 6-dimensional decomposable $K G$-module $V$, where $K$ has characteristic 2 and $G$ is the Klein four-group, such that $\mathscr{I}(L(V))$ is not finite (see Example 1).

\section{Free metabelian Lie algebras}

Let $G$ be any group, $K$ an arbitrary field and $V$ a $K G$-module. We identify $M^{1}(V)$ with $V$, so that $V$ is regarded as a subspace of $M(V)$. We note the standard fact that if $a_{1}, \ldots, a_{n} \in M(V)$ and $n \geq 3$, then the products $\left[a_{1}, \ldots, a_{n}\right]$ are symmetric with respect to the entries $a_{3}, \ldots, a_{n}$. If $\mathscr{V}$ is an ordered basis of $V$ then the products $\left[v_{1}, \ldots, v_{n}\right]$, where $n \geq 1, v_{1}, \ldots, v_{n} \in \mathscr{V}$ and $v_{1}>v_{2} \leq v_{3} \leq \cdots \leq v_{n}$, form a basis of $M(V)$ (see [3, Section 4.7]) and, for each $n$, those of degree $n$ form a basis of $M^{n}(V)$ which is called the standard basis of $M^{n}(V)$.

PROPOSITION 2.1. For a finite field $K$ and a finite group $G$, let $V$ be a 2-dimensional $K G$-module. Then $\mathscr{I}(M(V))$ is finite.

PROOF. In general, $M^{2}(V)$ is the exterior square of $V$. Since the left-normed metabelian Lie product $\left[v_{1}, \ldots, v_{n}\right]$ as $n$-variable function $V \times \cdots \times V \rightarrow M^{n}(V)$ is multilinear, alternating in the first two variables and symmetric in the others, the defining universal properties of exterior powers, symmetric powers and tensor products (see, for example, [8, Appendix B]) guarantee that there is a $K G$-homomorphism $M^{2}(V) \otimes S^{n-2}(V) \rightarrow M^{n}(V)$ whose image contains all the $\left[v_{1}, \ldots, v_{n}\right]$ and is therefore $M^{n}(V)$ itself. This much holds even if $\operatorname{dim} V>2$. Given that $\operatorname{dim} V=2$, dimension comparison shows that the surjective homomorphism in question is in fact an isomorphism. Further, since $M^{2}(V)$ is 1-dimensional, $M^{2}(V)^{*} \otimes M^{2}(V)$ (where 
$M^{2}(V)^{*}$ denotes the contragredient of $\left.M^{2}(V)\right)$ is the 1-dimensional trivial module, and therefore one has both

$$
M^{2}(V) \otimes S^{n-2}(V) \cong M^{n}(V) \text { and } \quad M^{2}(V)^{*} \otimes M^{n}(V) \cong S^{n-2}(V) .
$$

It follows that $X \mapsto M^{2}(V) \otimes X$ and $Y \mapsto M^{2}(V)^{*} \otimes Y$ provide a bijective correspondence between the set of the submodules $X$ of $S^{n-2}(V)$ and the set of the submodules $Y$ of $M^{n}(V)$, such that corresponding submodules are isomorphic. Thus $\mathscr{I}(M(V))$ is finite if and only if $\mathscr{I}(S(V))$ is finite.

Suppose now that $K$ is finite. Let $\Gamma=\mathrm{GL}(2, K), \Sigma=\operatorname{SL}(2, K)$, and let $U$ be the natural module for $K \Gamma$. It follows from Alperin and Kovács [1] that in this case $\mathscr{I}\left(S\left(\operatorname{Res}_{\Sigma} U\right)\right)$ is finite. Since the index $|\Gamma: \Sigma|$ is prime to the characteristic of $K$, any $K \Gamma$-module $W$ is a direct summand of the induced module $\operatorname{Ind}^{\Gamma} \operatorname{Res}_{\Sigma} W$ (see, for example, [2, Theorem 9.2]). In view of this, and of $S\left(\operatorname{Res}_{\Sigma} U\right)=\operatorname{Res}_{\Sigma} S(U)$, the finiteness of $\mathscr{I}(S(U))$ also follows.

It can be assumed without loss of generality that $G$ acts faithfully on $V$; equivalently, that $G$ is a subgroup of $\Gamma$ and $V=\operatorname{Res}_{G} U$. Since $S(V)=S\left(\operatorname{Res}_{G} U\right)=\operatorname{Res}_{G} S(U)$, we may now conclude that $\mathscr{I}(S(V))$ is finite, and hence so is $\mathscr{I}(M(V))$.

The homomorphism from $G$ to the trivial group $\{1\}$ extends to an algebra homomorphism $\varepsilon$ from $K G$ to the group algebra of $\{1\}$, which we may identify with the group algebra $K$. The kernel of $\varepsilon$ is the augmentation ideal $\Delta$ of $K G$ and so consists of all elements $\sum \alpha_{g} g$ of $K G$ with $\sum \alpha_{g}=0$. In particular, $\Delta$ is a submodule of $K G$. Moreover, the quotient $K G / \Delta$ is isomorphic with the trivial $K G$-module $K$. We write $V^{G}=\{v \in V: v g=v$ for all $g \in G\}$.

LEMMA 2.2. For all $n \geq 3$ there is a pair of $K G$-homomorphisms

$$
\phi_{n}: M^{n}(V) \rightarrow M^{3}(V) \otimes S^{n-3}(V), \quad \psi_{n}: M^{3}(V) \otimes S^{n-3}(V) \rightarrow M^{n}(V)
$$

such that the composite $\psi_{n} \phi_{n}$ is multiplication by $n(n-2)$ on $M^{n}(V)$.

Proof. By [9, Theorem 3.3], there is a pair of $K G$-homomorphisms

$$
\chi_{n, 3}: M^{n}(V) \rightarrow A^{3}(V) \otimes S^{n-3}(V), \quad \lambda_{n, 3}: A^{3}(V) \otimes S^{n-3}(V) \rightarrow M^{n}(V)
$$

such that the composite $\lambda_{n, 3} \chi_{n, 3}$ is multiplication by $n(n-2)$ on $M^{n}(V)$. The definition of the second homomorphism in [9] factors through $M^{3}(V) \otimes S^{n-3}(V)$, so the lemma follows.

COROLLARY 2.3. If $K$ is of characteristic 2 and $n$ is odd, then $M^{n}(V)$ is a direct summand of $M^{3}(V) \otimes S^{n-3}(V)$. 
Lemma 2.4. Let $\left\{x_{1}, \ldots, x_{m}, f\right\}=X \cup\{f\}$ be a basis of $V$, ordered by $x_{1}<\cdots<x_{m}<f$, and assume that $f \in V^{G}$. Then, for all $n \geq 3$, the map $u \mapsto[u, f]$, where $u \in M^{n-1}(V)$, is an injective $K G$-homomorphism $\mu_{n}$ : $M^{n-1}(V) \rightarrow M^{n}(V)$. Moreover, the elements

$$
\left[u_{1}, u_{2}, \ldots, u_{n}\right]+\operatorname{Im} \mu_{n} \quad\left(u_{i} \in X, u_{1}>u_{2} \leq \cdots \leq u_{n}\right)
$$

and

$$
\left[f, v_{1}, v_{2}, \ldots, v_{n-1}\right]+\operatorname{Im} \mu_{n} \quad\left(v_{i} \in X, v_{1} \leq v_{2} \leq \cdots \leq v_{n-1}\right)
$$

form a basis of the quotient $M^{n}(V) / \operatorname{Im} \mu_{n}$.

Proof. Since $f$ is fixed by $G$, the map $\mu_{n}$ agrees with the $G$-actions, and hence it is a $K G$-homomorphism. Moreover, $\mu_{n}$ maps the standard basis of $M^{n-1}(V)$ onto part of the standard basis of $M^{n}(V)$, and the remaining elements of the standard basis of $M^{n}(V)$ are precisely the ones listed in (2.1) and (2.2).

Now let $K$ be of characteristic $2, G=\left\langle\alpha, \beta \mid \alpha^{2}=\beta^{2}=(\alpha \beta)^{2}=1\right\rangle, V=\Delta$, and let $B_{n-1}$ denote the ( $\left.2 n-1\right)$-dimensional indecomposable $K G$-module with basis $y_{1}, \ldots, y_{n-1}, z_{0}, z_{1}, \ldots, z_{n-1}$ and $G$-action given by $z_{i} \alpha=z_{i} \beta=z_{i}, 0 \leq i \leq n-1$, $y_{i} \alpha=y_{i}+z_{i-1}$ and $y_{i} \beta=y_{i}+z_{i}, 1 \leq i \leq n-1$. Recall that $\Delta$ is the unique 3-dimensional indecomposable submodule of the regular $K G$-module.

THEOREM 2.5. For all odd $n$, with $n \geq 3, M^{n}(\Delta)$ is a free $K G$-module. For all even $n, M^{n}(\Delta)$ is isomorphic to a direct sum of (one copy of) $B_{n-1}$ and a free $K G$-module.

ProOF. The elements $u=1+\alpha, v=1+\beta$ and $f=1+\alpha+\beta+\alpha \beta$ form a basis of the module $\Delta$, and the action of $G$ on these basis elements is given by $u \alpha=u$, $u \beta=u+f, v \alpha=v+f, v \beta=v$ and $f \alpha=f \beta=f$. By Lemma 2.4, applied to $M^{n}(\Delta)$ and the basis $\{v, u, f\}$ of $\Delta$ with $v<u<f$, the quotient $M^{n}(\Delta) / \operatorname{Im} \mu_{n}$ has a basis consisting of the elements

and

$$
y_{i}=[u, \underbrace{v, \ldots, v}_{i}, \underbrace{u, \ldots, u}_{n-i-1}]+\operatorname{Im} \mu_{n} \quad(i=1, \ldots, n-1)
$$

$$
z_{i}=[f, \underbrace{v, \ldots, v}_{i}, \underbrace{u, \ldots, u}_{n-i-1}]+\operatorname{Im} \mu_{n} \quad(i=0, \ldots, n-1) .
$$

An easy calculation shows that in $M^{n}(\Delta) / \operatorname{Im} \mu_{n}$ the elements $z_{0}, \ldots, z_{n-1}$ are fixed by $G$, while $y_{i} \alpha=y_{i}+z_{i-1}$ and $y_{i} \beta=y_{i}+z_{i}(1 \leq i \leq n-1)$. Consequently,

$$
M^{n}(\Delta) / \operatorname{Im} \mu_{n} \cong B_{n-1}
$$


for all $n \geq 3$. One easily calculates that $M^{2}(\Delta) \cong B_{1}$ and $M^{3}(\Delta)$ is free of rank 2 . Since $M^{3}(\Delta)$ is free, so is the tensor product $M^{3}(\Delta) \otimes S^{n-3}(\Delta)$. By Corollary 2.3, for odd $n, M^{n}(\Delta)$ is a direct summand of $M^{3}(\Delta) \otimes S^{n-3}(\Delta)$, and hence it is also free. This proves the first part of the theorem. If $n \geq 4$ is even, then $\operatorname{Im} \mu_{n} \cong M^{n-1}(\Delta)$ is free, and since any free module is injective, (2.3) gives the second part of the theorem.

COROLLARY 2.6. Let $K$ be a finite field of characteristic 2, $U_{3}(K)$ the group of $3 \times 3$ upper unitriangular matrices over $K$ and $V$ the natural 3-dimensional $K U_{3}(K)$-module. Then $\mathscr{I}(M(V))$ is not finite.

ProOF. Let $g=\left(g_{i j}\right)$ and $h=\left(h_{i j}\right)$ be the elements of $U_{3}(K)$ for which $g_{12}=$ $g_{23}=0$ and $g_{13}=1$, while $h_{12}=h_{13}=0$ and $h_{23}=1$. It is easily seen that $G=\langle g, h\rangle$ is a Klein four-group such that $\operatorname{Res}_{G} V \cong \Delta$. Of course $\operatorname{Res}_{G} M(V)=M\left(\operatorname{Res}_{G} V\right)$, so if $\mathscr{I}(M(V))$ were finite, then so would be $\mathscr{I}\left(M\left(\operatorname{Res}_{G} V\right)\right)=\mathscr{I}(M(\Delta))$, contrary to Theorem 2.5.

\section{Free Lie algebras}

Let $G$ be any group and $K$ any field. A graded $K$-space is a $K$-space with a distinguished decomposition $V=V_{1} \oplus V_{2} \oplus \cdots$ where each $V_{n}$ is finite-dimensional. For each positive integer $n, L_{\text {grad }}^{n}(V)$ denotes the subspace of $L(V)$ spanned by all products $\left[v_{1}, v_{2}, \ldots, v_{k}\right]$, with $k \geq 1$, such that, for $i=1, \ldots, k, v_{i} \in V_{n(i)}$ for some $n(i) \geq 1$ with $n(1)+\cdots+n(k)=n$. In this way $L(V)$ becomes a graded $K$-space:

$$
L(V)=L_{\text {grad }}^{1}(V) \oplus L_{\text {grad }}^{2}(V) \oplus \cdots
$$

If $V$ is a finite-dimensional $K$-space regarded as a graded $K$-space with decomposition $V=V \oplus 0 \oplus 0 \oplus \cdots$, then $L_{\text {grad }}^{n}(V)=L^{n}(V)$ for all $n$. A graded $K G$-module is a $K G$ module $V$ with a distinguished decomposition $V=V_{1} \oplus V_{2} \oplus \cdots$ where each $V_{n}$ is a finite-dimensional $K G$-module. The homogeneous components $L_{\text {grad }}^{n}(V)$, as defined previously, are easily seen to be $K G$-submodules of $L(V)$. Thus $L(V)$ becomes a graded $K G$-module in the natural grading. We write $L_{\text {grad }}(V)$ instead of $L(V)$ when we work with a graded $K G$-module $V$.

Let $\left\{I_{\lambda}: \lambda \in \Lambda\right\}$ be a set consisting of one representative $I_{\lambda}$ from each isomorphism class of finite-dimensional indecomposable $K G$-modules, and consider a vector space $\Gamma_{K G}$ over the complex field $\mathbb{C}$ with this set as basis. For each finite-dimensional $K G$-module $V$, write [V] for the element $\sum \alpha_{\lambda} I_{\lambda}$ of $\Gamma_{K G}$ where each coefficient $\alpha_{\lambda}$ is the number of summands isomorphic to $I_{\lambda}$ in an unrefinable direct sum decomposition of $V$. Define a multiplication on $\Gamma_{K G}$ as the linear extension of $I_{\lambda} I_{\mu}=\left[I_{\lambda} \otimes I_{\mu}\right]$. Then $\Gamma_{K G}$ becomes a $\mathbb{C}$-algebra known as the Green algebra of $G$. 
We write $\Gamma_{K G}[[t]]$ for the algebra of all formal power series in an indeterminate $t$ with coefficients from $\Gamma_{K G}$, and $\Gamma_{K G}[[t]]^{\circ}$ for the ideal consisting of the power series with zero constant terms. For any graded $K G$-module $V$, we write

$$
[[V]]=\sum_{n \geq 1}\left[V_{n}\right] t^{n}
$$

This gives a convenient way of describing the graded isomorphism type of $V$. The map $V \mapsto[[V]]$ intertwines direct sums and tensor products of graded modules with addition and multiplication in $\Gamma_{K G}[[t]]$, and $\left[\left[L_{\mathrm{grad}}(V)\right]\right]=\sum_{n \geq 1}\left[L_{\mathrm{grad}}^{n}(V)\right] t^{n}$. Bryant (see [4]) studied functions $\Gamma_{K G}[[t]]^{\circ} \rightarrow \Gamma_{K G}[[t]]^{\circ}$ that always take [[V]] to $\left[\left[L_{\text {grad }}(V)\right]\right]$. To be able to quote one of his results, we need yet another definition.

For positive integers $r, s$, we define $w(r, s)$ by

$$
w(r, s)=\frac{1}{r+s} \sum_{d \mid(r, s)} \mu(d)\left(\begin{array}{c}
(r+s) / d \\
r / d
\end{array}\right),
$$

where $\mu$ is the Möbius function and the sum is over all positive integers $d$, which divide both $r$ and $s$. Note that the $w(r, s)$ are positive integers, because by Witt's formulae ([4, equation (3.6)]) they are dimensions of homogeneous components in free Lie algebras.

For the proof of the following result, we refer to [4, Theorem 2.4 and Theorem 4.2].

LEMMA 3.1. There is a function $\mathscr{L}_{K G}: \Gamma_{K G}[[t]]^{\circ} \rightarrow \Gamma_{K G}[[t]]^{\circ}$ such that

(I) for every graded $K G$-module $V$,

$$
\mathscr{L}_{K G}([[V]])=\left[\left[L_{\text {grad }}(V)\right]\right] ;
$$

(II) whenever $f_{1}, f_{2} \in \Gamma_{K G}[[t]]^{\circ}$,

$$
\mathscr{L}_{K G}\left(f_{1}+f_{2}\right)=\mathscr{L}_{K G}\left(f_{1}\right)+\mathscr{L}_{K G}\left(f_{2}\right)+\sum w(r, s) \mathscr{L}_{K G}\left(f_{1}^{r} f_{2}^{s}\right)
$$

where the summation is taken over all positive integers $r, s$.

Let $U$ and $V$ be any finite-dimensional $K G$-modules. For positive integers $r$ and $s$, we write $U^{r} V^{s}$ for the tensor product $U \otimes \cdots \otimes U \otimes V \otimes \cdots \otimes V$ where $U$ and $V$ are repeated $r$ and $s$ times, respectively. We write

$$
f_{1}=[[U]]=[U] t \text { and } f_{2}=[[V]]=[V] t .
$$

Note that $\left[L^{n}(U)\right]$ and $\left[L^{n}(V)\right]$ are the coefficients of $t^{n}$ in $\mathscr{L}_{K G}\left(f_{1}\right)$ and $\mathscr{L}_{K G}\left(f_{2}\right)$, respectively. For a positive integer $k$, let $\Theta^{k}: \Gamma_{K G}[[t]]^{\circ} \rightarrow \Gamma_{K G}[[t]]^{\circ}$ be the substitution of $t^{k}$ for $t$. It has been noted in [4, page 181] that $\Theta^{k} \circ \mathscr{L}_{K G}=\mathscr{L}_{K G} \circ \Theta^{k}$ for 
$k \geq 1$. It is now easy to see that

$$
\mathscr{L}_{K G}\left(f_{1}^{r} f_{2}^{s}\right)=\Theta^{r+s}\left(\mathscr{L}_{K G}\left(\left[U^{r} V^{s}\right] t\right)\right)=\sum_{n \geq 1}\left[L^{n}\left(U^{r} V^{s}\right)\right] t^{n(r+s)}
$$

By Lemma 3.1 and equation (3.1), we obtain that

$$
L(U \oplus V) \cong L(U) \oplus L(V) \oplus \bigoplus_{T} L(T),
$$

where $T$ ranges through all tensor products $U^{r} V^{s}$ with $r, s$ positive integers, taking each such value at least once, but many of them more than once. We write $\mathscr{T}$ for the set of the aforementioned tensor products. The following result has been proved in [6, Theorem 4.1].

LEMMA 3.2. Let $G$ be any group, $K$ any field and $V$ any finite-dimensional $K G$-module. Then for any positive integer $n, L^{n}(V)$ is isomorphic to a direct sum of modules, each of which has the form $L^{k}(W)$ for some divisor $k$ of $n$ and some indecomposable direct summand $W$ of $A^{n / k}(V)$.

If $T$ is an element of $\mathscr{T}$, then so is each tensor power $A^{n / k}(T)$ of $T$. Thus if $W$ is an indecomposable direct summand of some $A^{n / k}(T)$, then $W$ is a direct summand of some element of $\mathscr{T}$. By Lemma 3.2, we conclude that

$$
\bigcup_{T \in \mathscr{T}} \mathscr{I}(L(T)) \subseteq \bigcup_{W \in \mathscr{W}} \mathscr{I}(L(W)),
$$

where $\mathscr{W}=\bigcup_{T \in \mathscr{T}} \mathscr{I}(T)$. Conversely, the Elimination Theorem (see [5, Lemma 2.2]) shows that if $W \in \mathscr{I}(T)$ then $L(W)$ is a direct summand of $L(T)$, and so $\mathscr{I}(L(W)) \subseteq$ $\mathscr{I}(L(T))$. Therefore, by (3.2), we obtain that

$$
\mathscr{I}(L(U \oplus V))=\mathscr{I}(L(U)) \cup \mathscr{I}(L(V)) \cup \bigcup_{W \in \mathscr{W}} \mathscr{I}(L(W)) .
$$

Suppose now that $U$ and $V$ are graded modules that are concentrated in degrees $k$ and $\ell$, respectively (so $U=U_{k}$ while $U_{i}=0$ if $i \neq k$, and $V=V_{\ell}$ while $V_{j}=0$ if $j \neq \ell$ ). Repeating the previous argument with $f_{1}=[[\dot{U}]]=[U] t^{k}$ and $f_{2}=[[V]]=$ $[V] t^{\ell}$ yields first a version of (3.1) in which both occurrences of $r+s$ are replaced by $r k+s \ell$, but then proceeds as before; the only change being required in (3.2) and (3.3) is to replace each $L$ by $L_{\text {grad }}$. On the right hand side of the version of (3.3) so obtained, we prefer to retain 'the other' gradings. To this end, we note that $L^{r}(U)=L_{\text {grad }}^{r k}(U)$, and so on: if $W$ is a direct summand of $U^{r} V^{s}$, then $L^{m}(W)=L_{\mathrm{grad}}^{m(r k+s \ell)}(W)$. In these terms, the conclusions may be put as follows. 
PROPOSITION 3.3. Let $K$ be any field, $G$ any group, and $U, V$ graded $K G$-modules concentrated in degrees $k, \ell$, respectively. For each positive integer $q$,

$$
\mathscr{I}\left(L_{\mathrm{grad}}^{q}(U \oplus V)\right)=\mathscr{I}\left(L^{q / k}(U)\right) \cup \mathscr{I}\left(L^{q / \ell}(V)\right) \cup \bigcup_{d \mid q} \bigcup_{W} \mathscr{I}\left(L^{q / d}(W)\right),
$$

where Lie powers to non-integers are read as 0 , the range of $d$ is the set of positive divisors of $q$, and $W$ runs over the union of the $\mathscr{I}\left(U^{r} V^{s}\right)$ with $r, s \geq 1$ and $r k+s \ell=d$.

For the proof of the following result, we refer to [12, Section 2].

LEMMA 3.4. Let $K$ be a field of characteristic 2 and $V$ any 2-dimensional $K$ space. Let $\{x, y\}$ be a $K$-basis of $V$ and let $\mathscr{E}$ be the subset of $R(V)$ defined by $\mathscr{E}=\left\{x^{2},[x, y], y^{2},[x, y, x],[x, y, y]\right\}$. Then the restricted Lie subalgebra $E^{*}$ of $R(V)$ generated by $\mathscr{E}$ is free on $\mathscr{E}$.

Let $E$ be the Lie subalgebra of $E^{*}$ generated by $\mathscr{E}$. It is a direct consequence of Lemma 3.4 that $E$ is freely generated by $\mathscr{E}$. Since $\mathscr{E}$ is a $K$-basis of $R^{2}(V) \oplus R^{3}(V)$, we may write $E=L\left(R^{2}(V) \oplus R^{3}(V)\right)$.

THEOREM 3.5. Let $K$ be a field of characteristic 2, $G$ any group and $V$ any 2-dimensional $K G$-module. Then $L^{n}(V)=L_{\text {grad }}^{n}\left(R^{2}(V) \oplus R^{3}(V)\right)$ for $n \geq 3$.

ProOF. Since $\left[a, b^{2}\right]=[a, b, b]$ for all $a, b \in A(V)$, we obtain that $\left[a, b^{2}\right] \in L(V)$ for all $a, b \in R(V)$ and so it is easily verified that

$$
\left.L_{\text {grad }}^{n}\left(R^{2}(V) \oplus R^{3}(V)\right)\right) \subseteq L^{n}(V) \text { for all } n \geq 3 .
$$

Next, we shall use induction on $n$ to show that

$$
L^{n}(V) \subseteq L_{\text {grad }}^{n}\left(R^{2}(V) \oplus R^{3}(V)\right) \text { for all } n \geq 2 .
$$

For $n=2$ and $n=3$, our claim is trivially true, so we may assume that $n \geq 4$. Let $\{x, y\}$ be a $K$-basis of $V$. To prove our claim, it is enough to show that $\left[x_{1}, \ldots, x_{n}\right] \in$ $L_{\text {grad }}^{n}\left(R^{2}(V) \oplus R^{3}(V)\right)$ whenever $x_{1}=x, x_{2}=y$ and $x_{3}, \ldots, x_{n} \in\{x, y\}$. Our inductive hypothesis implies that $L^{n}(V) \cap L(V)^{\prime \prime} \subseteq L_{\text {grad }}^{n}\left(R^{2}(V) \oplus R^{3}(V)\right)$ and so, working modulo $L^{n}(V) \cap L(V)^{\prime \prime}$, we obtain that

$$
\left[x_{1}, \ldots, x_{n}\right]=[x, y, \underbrace{y, \ldots, y}_{r}, \underbrace{x, \ldots, x}_{s}]+w
$$

for some $w \in L^{n}(V) \cap L(V)^{\prime \prime}$ and some $r, s$ with $r+s=n-2$. Since $K$ has characteristic 2 , we have

$$
[a, \underbrace{b, \ldots, b}_{2 m}]=[a, \underbrace{b^{2}, \ldots, b^{2}}_{m}]
$$


for all non-negative integers $m$ and all $a, b \in A(V)$. Using this repeatedly, we see that

$$
\begin{aligned}
& {[x, y, \underbrace{y, \ldots, y}_{2 k}, \underbrace{x, \ldots, x}_{2 \ell}]=[[x, y], \underbrace{y^{2}, \ldots, y^{2}}_{k}, \underbrace{x^{2}, \ldots, x^{2}}_{\ell}]+w_{1},} \\
& {[x, y, \underbrace{y, \ldots, y}_{2 k}, \underbrace{x, \ldots, x}_{2 \ell+1}]=[\left[x^{2}, y\right], \underbrace{y^{2}, \ldots, y^{2}}_{k}, \underbrace{x^{2}, \ldots, x^{2}}_{\ell}]+w_{2},} \\
& {[x, y, \underbrace{y, \ldots, y}_{2 k+1}, \underbrace{x, \ldots, x}_{2 \ell}]=[\left[x, y^{2}\right], \underbrace{y^{2}, \ldots, y^{2}}_{k}, \underbrace{x^{2}, \ldots, x^{2}}_{\ell}]+w_{3},} \\
& {[x, y, \underbrace{y, \ldots, y}_{2 k+1}, \underbrace{x, \ldots, x}_{2 \ell+1}]=[\left[x^{2}, y^{2}\right], \underbrace{y^{2}, \ldots, y^{2}}_{k}, \underbrace{x^{2}, \ldots, x^{2}}_{\ell}]+w_{4},}
\end{aligned}
$$

where $w_{1}, \ldots, w_{4}$ lie in $L^{n}(V) \cap L(V)^{\prime \prime}$ and hence are contained in $L\left(R^{2}(V) \oplus R^{3}(V)\right)$. This proves statement (3.5) and so completes the proof of the theorem.

Let $G$ be the Klein four-group and $C$ a faithful 2-dimensional $K G$-module, with $K$ a field of characteristic 2 . For such a module to exist, $K$ must have more than 2 elements: one can see from Conlon [7] that there exists precisely one isomorphism type for each element of $K$ different from 0 and 1 . Let $D$ be the regular $K G$-module, $B$ the unique 3-dimensional quotient of $D$, and $A$ the 1-dimensional trivial $K G$-module. It is easy to see that $L^{2}(C) \cong A, R^{2}(C) \cong B$ and $L^{3}(C)=R^{3}(C) \cong C$. The multiplication rules given by Conlon [7, page 89] yield that, for $m, n \geq 1, \mathscr{I}\left(B^{m} C^{n}\right)$ is either $\{C, D\}$ or $\{C \otimes C, D\}$, depending on whether $n$ is odd or even. From Theorem 3.5 and Proposition 3.3 we now get that, for $q \geq 4$,

$$
\mathscr{I}\left(L^{q}(C)\right)=\mathscr{I}\left(L^{q / 2}(B)\right) \cup \mathscr{I}\left(L^{q / 3}(C)\right) \cup \bigcup_{d \mid q} \bigcup_{W} \mathscr{I}\left(L^{q \mid d}(W)\right)
$$

where the range of $W$ is the union of the $\mathscr{I}\left(B^{m} C^{n}\right)$ with $m, n \geq 1$ and $2 m+3 n=d$. This range is readily seen to be empty when $d \leq 4$ or $d=6$, it is $\{C, D\}$ when $d$ is an odd number greater than 3 , and it is $\{C \otimes C, D\}$ when $d$ is an even number greater than 6. Equivalently, if $q \geq 4$, then

$$
\begin{aligned}
\mathscr{I}\left(L^{q}(C)\right)= & \mathscr{I}\left(L^{q / 2}(B)\right) \cup \bigcup_{r} \mathscr{I}\left(L^{q / r}(C)\right) \\
& \cup \bigcup_{s} \mathscr{I}\left(L^{q / s}(C \otimes C)\right) \cup \bigcup_{t} \mathscr{I}\left(L^{q / t}(D)\right),
\end{aligned}
$$

where $r$ is odd and $r \geq 3, s$ is even and $s \geq 8$, and either $t=5$ or $t \geq 7$, and $r, s, t$ range through the divisors of $q$ subject only to these conditions. It follows immediately that $\mathscr{I}(L(C)) \supseteq \mathscr{I}\left(L(B)^{\prime}\right) \cup \mathscr{I}(L(C \otimes C)) \cup \mathscr{I}(L(D))$, where $L(B)^{\prime}$ is the derived algebra of $L(B)$. Let $X$ be any indecomposable in $\mathscr{I}(L(C))$, and choose $q$ minimal with respect to $X \in \mathscr{I}\left(L^{q}(C)\right)$. If $q \geq 4$, we may apply (3.6); by the minimality of $q$, 
no $\mathscr{I}\left(L^{q / r}(C)\right)$ can contain $X$, so $X$ must lie in $\mathscr{I}\left(L(B)^{\prime}\right) \cup \mathscr{I}(L(C \otimes C)) \cup \mathscr{I}(L(D))$. In view of $L^{3}(C) \cong L^{1}(C)=C$ and $L^{2}(C) \cong A$, we have proved the following relation $\mathscr{I}(L(C))=\{A, C\} \cup \mathscr{I}\left(L(B)^{\prime}\right) \cup \mathscr{I}(L(C \otimes C)) \cup \mathscr{I}(L(D))$.

THEOREM 3.6. Let $G$ be the Klein four-group, $K$ a field of characteristic 2 containing more than two elements and $C$ any faithful 2-dimensional $K G$-module. Let $D$ be the regular $K G$-module, $B$ the unique 3-dimensional quotient of $D$ and $A$ the 1-dimensional trivial $K G$-module. Then, $\mathscr{I}(L(C))$ is finite if and only if $\mathscr{I}(L(B))$, $\mathscr{I}(L(D))$ and $\mathscr{I}(L(C \otimes C))$ are finite.

EXAMPLE 1 (Michos). Let $K$ be a field of characteristic 2 and $G$ a Klein four-group. Conlon [7] described an infinite sequence $A_{1}, A_{2}, \ldots$ of indecomposable $K G$-modules such that $A_{1}$ is the augmentation ideal of $K G, \operatorname{dim} A_{n}=2 n+1$, and $A_{r+s}$ is a direct summand of $A_{r} \otimes A_{s}$. An easy induction on $n$ shows that therefore $A_{n}$ is a direct summand of the $n$-fold tensor power of $A_{1}$. By (3.2) with $U=V=A_{1}$, it follows that each $A_{n}$ is a direct summand of $L\left(A_{1} \oplus A_{1}\right)$, and so $\mathscr{I}\left(L\left(A_{1} \oplus A_{1}\right)\right)$ is infinite.

\section{Acknowledgements}

I wish to thank Professor L. G. Kovács for his valuable suggestions in an earlier version of this manuscript. Namely, the proof of Proposition 3.3 has been suggested by him simplifying our original proof. Also, I would like to thank Professor R. M. Bryant for the constructive discussions on this subject. Finally, I would like to thank the referee who suggested a number of improvements.

\section{References}

[1] J. Alperin and L. G. Kovács, 'Periodicity of Weyl modules for SL(2,q)', J. Algebra 74 (1982), 52-54.

[2] J. L. Alperin, Local representation theory, Cambridge Studies in Advanced Mathematics 11 (Cambridge University Press, Cambridge, 1993).

[3] Yu. A. Bakhturin, Identical relations in Lie algebras (Nauka, Moscow, 1985), in Russian; English translation: (VNU Science Press, Utrecht, 1987).

[4] R. M. Bryant, 'Free Lie algebras and formal power series', J. Algebra 253 (2002), 167-188.

[5] R. M. Bryant, L. G. Kovács and R. Stöhr, 'Lie powers of modules for groups of prime order', Proc. London Math. Soc. (3) 84 (2002), 343-374.

[6] _ - 'Lie powers of modules for GL(2, p)', J. Algebra 260 (2003), 617-630.

[7] S. B. Conlon, 'Certain representation algebras', J. Aust. Math. Soc. 5 (1965), 83-99.

[8] W. Fulton and J. Harris, Representation theory: a first course (Springer, New York, 1991).

[9] T. Hannebauer and R. Stöhr, 'Homology of groups with coefficients in free metabelian Lie powers and exterior powers of relation modules and applications to group theory', in: Proceedings of the 
Second International Group Theory Conference (Bressanone, 1989), Rend. Circ. Mat. Palermo (2) Suppl. 23 (1990), 77-113.

[10] B. Huppert and N. Blackburn, Finite groups II (Springer, Berlin, 1982).

[11] D. Karagueuzian and P. Symonds, 'The module structure of a group action on a polynomial ring', J. Algebra 218 (1999), 672-692.

[12] R. Stöhr, 'Restricted Lazard elimination and modular Lie powers', J. Aust. Math. Soc. 71 (2001), 259-277.

\section{Faculty of Sciences}

Department of Mathematics

Aristotle University of Thessaloniki

GR 541 24, Thessaloniki

Greece

e-mail: apapist@math.auth.gr 